\title{
Quantum entanglement and information processing via excitons in optically-driven quantum dots
}

\author{
John H. Reina ${ }^{1 \aleph}$, Luis Quiroga ${ }^{2 \dagger}$, and Neil F. Johnson ${ }^{1 \ddagger}$ \\ ${ }^{1}$ Physics Department, Clarendon Laboratory, Oxford University, Oxford, OX1 3PU, England \\ ${ }^{2}$ Departamento de Física, Universidad de Los Andes, Santafé de Bogotá, A.A. 4976, Colombia
}

\begin{abstract}
We show how optically-driven coupled quantum dots can be used to prepare maximally entangled Bell and Greenberger-Horne-Zeilinger states. Manipulation of the strength and duration of the selective light-pulses needed for producing these highly entangled states provides us with crucial elements for the processing of solid-state based quantum information. Theoretical predictions suggest that several hundred single quantum bit rotations and Controlled-Not gates could be performed before decoherence of the excitonic states takes place.
\end{abstract}

PACS number(s): 03.67.-a, 71.10.Li, 71.35.-y, 73.20.Dx

\section{INTRODUCTION}

Quantum computation, quantum communication, quantum cryptography and quantum teleportation [1 15$]$ are some of the most exciting applications of the fundamental principles of quantum theory. Since the seminal idea of Feynman in 1982 [1] and the work of Deutsch in 1985 [2], both pure and applied research in the field of quantum information processing has blossomed. In 1994, Shor [6] opened the way to new fast quantum searching algorithms: he discovered that a quantum computer can factorize large integers. Two years later arrived the proof that quantum error-correcting codes exist [7]. Up until now, such quantum mechanical computers have been proposed in terms of trapped ions and atoms [8], cavity quantum electrodynamics (QED) [9], nuclear magnetic resonance [10], Josephson junctions [1] and semiconductor nanostructures [12] schemes. All of the above proposals have decoherence and operational errors as the main obstacles for their experimental realization, which pose much stronger problems here than in classical computers.

There is much current excitement about the possibility of using solid-state based devices for the achievement of quantum computation tasks. In particular, semiconductor nanostructure fabrication technology is well developed and hence offers us a wide and promising arena for the challenging project of building quantum information processors. Because of their quantum mechanical nature and their potential scalability properties, semiconductor quantum dots (QDs) are very promising candidates for the implementation of quantum computing processes. Several solid state design schemes for quantum computation have been proposed to date: Kane [12] has proposed a scheme which encodes information onto the nuclear spins of donor atoms in doped silicon electronic devices where externally applied electric fields are used to perform logical operations on individual spins. Loss and DiVincenzo [12] have presented a scheme based on elec- tron spin effects, in which coupled quantum dots are used as a quantum gate. This scheme is based on the fact that the electron spins on the dots have an exchange interaction $J$ which changes sign with increasing external magnetic field. Possible quantum gate implementations have also been proposed by Barenco et al. [12] by considering electronic charge effects in coupled QDs, however this scheme has as the main disadvantage rapid phonon decoherence, as compared with the above proposals. More recently, Imamoglu et al. 12] have considered a quantum computer model based on both electron spins and cavity QED which is capable of realizing controlled interactions between two distant QD spins. In their model, the effective long-range interaction is mediated by the vacuum field of a high finesse microcavity, and single quantum bit (qubit) rotations and Controlled-Not (CNOT) operations are realized using electron-hole Raman transitions induced by classical laser fields and the cavity mode. Vrijen et al. [12] considered electron spin resonance transistors in Silicon-Germanium heterostructures: one and two qubit operations are performed by applying a gate bias.

In this paper we focus on optically-driven coupled QDs in order to obtain highly entangled states of excitons and hence provide a mechanism for processing quantum information over a reasonable parameter window, before decoherence of the excitonic states takes place. In the physical implementation of the quantum entanglement scheme proposed here, we exploit recent experimental results involving coherent optical control of excitons in single quantum dots on the nanometer and femtosecond scales 13,15 . The amazing degree of control of the quantum states of these individual "artificial atoms" 13. due to the manipulation of the confined state wave function of a single dot is an exciting and promising development. As one exciton can be trapped in the dot, we have the direct possibility to use QDs as elements with quantum memory capacity in quantum computation operations, through a precise and controlled excitation of the system. As demonstrated in [13], it is possible to excite and 
probe only one individual QD within a broad distribution of dots, with the important result that the dephasing time is much longer in a single dot (40ps) than in the bulk semiconductors $(<1 \mathrm{ps})$ studied before. Hence, new experimental work means that much longer intrinsic coherence times are currently available, a fact of fundamental importance when looking towards the practical implementation of single qubit rotations and quantum CNOT gates which are crucial elements for carrying out quantum information processing tasks.

The outline of this paper is as follows: Section II gives a detailed description of the coupling of qubits (i.e. QDs) to pulses of light and the generation of maximally entangled Bell (or EPR) [16,17 and Greenberger-HorneZeilinger (GHZ) 18 states in systems comprising two and three quantum dots, respectively. The discussion of the results and experimental considerations are presented in Section III. In Section IV we review the elements required for performing quantum computation tasks and the links with the solid state set-up proposed here. Conclusions are given in Section V.

\section{II. \\ GENERATION OF MAXIMALLY ENTANGLED STATES IN OPTICALLY-DRIVEN QUANTUM DOTS}

Quantum entanglement is of fundamental interest since many of the most basic aspects of quantum theory require its successful generation and manipulation. In particular Bell and GHZ maximally entangled states are the starting point for fundamental discussions such as the violation of Bell's inequalities [19] and the nonlocality problem [17], as well as for teleportation [5] and

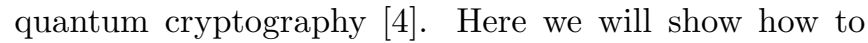
generate maximally entangled states of two and three qubits of the form $\left|\Psi_{\text {Bell }}\right\rangle=\frac{1}{\sqrt{2}}\left(|00\rangle+e^{i \varphi}|11\rangle\right)$ and $\left|\Psi_{G H Z}\right\rangle=\frac{1}{\sqrt{2}}\left(|000\rangle+e^{i \varphi}|111\rangle\right)$, for arbitrary values of the phase factor $\varphi$ [20], using a semiconductor nanostructure set-up. We consider a system of $N$ identical and equispaced QDs, containing no net charge, which are radiated by long-wavelength classical light. Hence formation of single excitons within the individual QDs and their inter-dot transfer can be described in the frame of the rotating wave approximation (RWA), by the Hamiltonian 21,22 $(\hbar=1)$ :

$$
\begin{array}{r}
H(t)=\frac{\epsilon}{2} \sum_{p=1}^{N}\left\{c_{p}^{\dagger} c_{p}-h_{p} h_{p}^{\dagger}\right\}+\frac{W}{2} \sum_{p, p^{\prime}=1}^{N}\left\{c_{p}^{\dagger} h_{p^{\prime}} c_{p^{\prime}} h_{p}^{\dagger}+\right. \\
\left.h_{p} c_{p^{\prime}}^{\dagger} h_{p^{\prime}}^{\dagger} c_{p}\right\}+\xi(t) \sum_{p=1}^{N} c_{p}^{\dagger} h_{p}^{\dagger}+\xi^{*}(t) \sum_{p=1}^{N} h_{p} c_{p},
\end{array}
$$

where $c_{p}^{\dagger}\left(h_{p}^{\dagger}\right)$ is the electron (hole) creation operator in the $p$ th quantum dot, $\epsilon$ is the $\mathrm{QD}$ band gap, $W$ the interdot interaction, and $\xi(t)$ the laser pulse shape. The operators involved in Eq. (1) obey the anticommutation rules $\left\{c_{p^{\prime}}, c_{p}^{\dagger}\right\}=\left\{h_{p^{\prime}}, h_{p}^{\dagger}\right\}=\delta_{p p^{\prime}}$. By introducing the new operators

$$
\begin{array}{r}
J_{+}=\sum_{p=1}^{N} c_{p}^{\dagger} h_{p}^{\dagger}, J_{-}=\sum_{p=1}^{N} h_{p} c_{p}, \\
J_{Z}=\frac{1}{2} \sum_{p=1}^{N}\left\{c_{p}^{\dagger} c_{p}-h_{p} h_{p}^{\dagger}\right\},
\end{array}
$$

Eq. (1) adopts the form

$$
H(t)=H_{0}+H_{L}(t)
$$

where

$$
H_{0}=\epsilon J_{Z}+W\left(J^{2}-J_{Z}^{2}\right), H_{L}(t)=\xi(t) J_{+}+\xi^{*}(t) J_{-},
$$

with $J^{2} \equiv \frac{1}{2}\left[J_{+} J_{-}+J_{-} J_{+}\right]+J_{Z}^{2}$. The $J_{i}$-operators obey the usual angular momentum commutation relations $\left[J_{Z}, J_{ \pm}\right]= \pm J_{ \pm},\left[J_{+}, J_{-}\right]=2 J_{Z}$, and $\left[J^{2}, J_{+}\right]=$ $\left[J^{2}, J_{-}\right]=\left[J^{2}, J_{Z}\right]=0$. In going from Eq. (1) to Eq. (3) we have switched from a dot-selective (index $p$ ) to a non-selective description. Since the dots are equidistant from each other, this description is appropriate for up to 4 dots (placed at the corners of a tetrahedron). Hence the quantum dynamical problem associated with the time evolution of any initial state under the action of $H(t)$ is described by:

$$
i \partial_{t}|\Psi(t)\rangle_{S}=\left\{H_{0}+H_{L}(t)\right\}|\Psi(t)\rangle_{S},
$$

where the subscript $S$ indicates Schrödinger picture. We consider the laser pulse shape $\xi(t)=A e^{-i \omega t}$, where $A$ gives the electron-photon coupling and the incident electric field strength. We also introduce the unitary transformation $\Lambda(t)=e^{-i \omega J_{Z} t}$, whose application in the Schrödinger picture leads us from the laboratory frame (LF) to the rotating frame (RF) using the rule $|\Psi(t)\rangle_{\Lambda}=\Lambda^{\dagger}(t)|\Psi(t)\rangle_{S}$. Hence Eq. (5) may be rewritten as:

$$
i \partial_{t}|\Psi(t)\rangle_{\Lambda}=H^{\prime}|\Psi(t)\rangle_{\Lambda}
$$

with

$$
H^{\prime}=\Delta_{\omega} J_{Z}+W\left(J^{2}-J_{Z}^{2}\right)+A J_{+}+A^{*} J_{-} .
$$

Here $\Delta_{\omega} \equiv \epsilon-\omega$ is the detuning parameter. We note the importance of the $\Lambda$-transformation: the new Hamiltonian $H^{\prime}$ is time-independent. From a practical point of view, parameters $A$ and $\Delta_{\omega}$ are adjustable in the experiment to give control over the system of QDs (or qubits). Since $J^{2}$ commutes with the operators $J_{ \pm}, H^{\prime}$ may be diagonalized separately in each one 
of these $J$-subspaces. Consider the $\{J, q\}$ subspace spanned by $|M\rangle \equiv|J, M ; q\rangle$ : the only possible values for $J$ are $\frac{N}{2}, \frac{N}{2}-1, \ldots, \frac{1}{2}$ or 0 , and for each $J$-fixed value, we have $2 J+1$ different values for $M$, which are given by $M=-\frac{N}{2},-\frac{N}{2}+1, \ldots, \frac{N}{2}-1, \frac{N}{2}$. We introduce the label $q$ to further distinguish the states: $q=1,2, \ldots, D_{J}$, where the multiplicity $D_{J}$, i.e. the number of states having angular momentum $J$ and $M=J$, is given by $D_{J}=\frac{2 J+1}{J+\frac{N}{2}+1}\left(\begin{array}{c}N \\ \frac{N}{2}+J\end{array}\right)$. The product states $\prod_{k=1}^{N}\left|m_{k}\right\rangle \equiv\left|m_{1}, \ldots, m_{N}\right\rangle$, with $J_{Z}=\sum_{k} m_{k}$ form a $2^{N}$-dimensional basis which span the Hilbert space $S U(2)^{\otimes N}$. In this basis, the $2^{N}$ eigenvalues of $H^{\prime}$ are obtained by diagonalizing the Hamiltonian matrix of elements $\left\langle J, M, q\left|H^{\prime}\right| J^{\prime}, M^{\prime}, q^{\prime}\right\rangle$. We get the non-zero elements as follows:

$$
\begin{aligned}
\left\langle M\left|H^{\prime}\right| M^{\prime}\right\rangle & =\left(\Delta_{\omega} M+W\left[J(J+1)-M^{2}\right]\right) \delta_{M, M^{\prime}}+ \\
& A \sqrt{J(J+1)-M^{\prime}\left(M^{\prime}+1\right)} \delta_{M, M^{\prime}+1}+ \\
& A^{*} \sqrt{J(J+1)-M^{\prime}\left(M^{\prime}-1\right)} \delta_{M, M^{\prime}-1} .
\end{aligned}
$$

The matrix elements given in Eq. (8) provide us with the general rule for any number of QDs. Since the right side of this equation does not depend on $q$ we only need to diagonalize a square matrix of side $2 J+1$ for each $J$. Every eigenvalue so obtained occurs $D_{J}$ times in the entire spectrum. Next, we show that solving the eigenfunction problem associated with Eq. (8) leads to the generation of highly $N$-entangled states of excitons in QDs.

\section{A. Coupling of $\mathrm{N}=2$ QDs and generation of Bell states}

In this section we describe the procedure for the generation of entangled Bell states $\left|\Psi_{\text {Bell }}\right\rangle=\frac{1}{\sqrt{2}}(|00\rangle+$ $\left.e^{i \varphi}|11\rangle\right)$ for arbitrary values of the phase factor $\varphi$. Here 0 (1) denotes a zero-exciton (single exciton) QD, and the direct product of the quantum states $|j k\rangle \equiv|j\rangle \otimes|k\rangle$ form a four-dimensional basis in the Hilbert space $S U(2) \otimes$ $S U(2)$. In the $J=1$ subspace [23], $M \equiv\{-1,0,1\}$. We define $\left|M_{1}\right\rangle \equiv|J=1, M=-1\rangle \equiv|0\rangle,\left|M_{2}\right\rangle \equiv$ $|J=1, M=0\rangle \equiv|1\rangle$, and $\left|M_{3}\right\rangle \equiv|J=1, M=1\rangle \equiv|2\rangle$, as the vacuum of excitons, the single-exciton state and the biexciton state respectively. In the absence of light, we have:

$$
E(J, M)=\Delta_{\omega} M+W\left[J(J+1)-M^{2}\right],
$$

so the energy levels of the system are $E_{0} \equiv E(1,-1)=$ $W-\Delta_{\omega}, E_{1} \equiv E(1,0)=2 W$, and $E_{2} \equiv E(1,1)=$ $W+\Delta_{\omega}$. Note that $E_{2,0} \equiv E_{2}-E_{0}=2 \Delta_{\omega}$ is unaffected by the interdot interaction strength $W$. Next, consider the action of the radiation pulse $\xi(t)$ over this pair of qubits; in the $J=1$ subspace the Hamiltonian adopts the simple form:

$$
\widehat{H^{\prime}}=\left(\begin{array}{ccc}
W-\Delta_{\omega} & \sqrt{2} A^{*} & 0 \\
\sqrt{2} A & 2 W & \sqrt{2} A^{*} \\
0 & \sqrt{2} A & W+\Delta_{\omega}
\end{array}\right),
$$

where $A \equiv|A| e^{i \phi}$ defines the real amplitude and the phase of the electron-photon coupling. Diagonalization gives the eigenenergies and eigenfunctions associated with Eq. (10). We get

$$
\begin{aligned}
& E^{3}-4 W E^{2}+\left(5 W^{2}-4|A|^{2}-\Delta_{\omega}^{2}\right) E+ \\
& 2 W\left(\Delta_{\omega}^{2}+2|A|^{2}-W^{2}\right)=0,
\end{aligned}
$$

as the eigenenergy equation. In resonance $\Delta_{\omega} \equiv 0$, hence we see that Eq. (11) has solutions:

$$
E_{0}=W, \text { and } E_{1,2}=\frac{1}{2}\left(3 W \pm \sqrt{16|A|^{2}+W^{2}}\right) .
$$

The eigenenergies for the case of an off-resonance pulse of light can also be found analytically [24]. We do not give the explicit expressions for brevity. The eigenfunctions for the $N=2$ problem are given by:

$$
\begin{gathered}
\left|E=E_{k}\right\rangle=\Gamma_{k} \times \\
\left(|0\rangle+\frac{E_{k}+\Delta_{\omega}-W}{\sqrt{2}|A|}|1\rangle-\frac{2|A|^{2}+\left(2 W-E_{k}\right)\left(E_{k}+\Delta_{\omega}-W\right)}{2|A|^{2}}|2\rangle\right),
\end{gathered}
$$

where

$\Gamma_{k}=\sqrt{2}|A|\left[4|A|^{2}+\left(\Delta_{\omega}+W\right)\left(E_{k}+\Delta_{\omega}-W\right)\right]^{-\frac{1}{2}}$, with $E_{k}$ given as above $(k=0,1,2)$. The procedure described here enables us to perform the calculation of the laser pulse length required for generating the searched entangled Bell states.

In general, for any value of $N$, the total wave function associated with the initial condition $|\Psi(t=0)\rangle=\left|\Psi_{0}\right\rangle$ can be expressed as $|\Psi(t)\rangle_{\Lambda}=\sum_{k} C_{k} e^{-i E_{k} t}\left|\psi_{k}\right\rangle$, where $H^{\prime}\left|\psi_{k}\right\rangle=E_{k}\left|\psi_{k}\right\rangle$, and $\left|\psi_{k}\right\rangle=\sum_{j} A_{k j}\left|M_{j}\right\rangle$. Here the normalization coefficients $C_{k}$ [25] depend on the chosen initial condition $\left|\Psi_{0}\right\rangle$. The matrix elements $A_{k j}$ must be determined for each particular value of $N$, and $\left|M_{j}\right\rangle \equiv$ $\left|J, M_{j} ; q\right\rangle$ as indicated earlier in this section. Hence, the total wave function $|\Psi(t)\rangle_{\Lambda}$ can be written as:

$$
|\Psi(t)\rangle_{\Lambda}=\sum_{k} \sum_{j} C_{k} A_{k j} e^{-i E_{k} t}\left|M_{j}\right\rangle .
$$

For the case of $N=2$ QDs, it is a straightforward exercise to compute the explicit coefficients of Eq. (14) for both of the $J$-subspaces that span the Hilbert space $S U(2) \otimes S U(2)$. Next we centre our attention on the discussion of finding the conditions to produce the maximally entangled Bell states. To achieve this, we project the state $\left|\Psi_{\text {Bell }}\right\rangle$ over the wave function given by Eq. (14) obtaining the result:

$$
\left\langle\Psi_{\text {Bell }} \mid \Psi(t)\right\rangle_{\Lambda}=\frac{1}{\sqrt{2}} \sum_{k} C_{k}\left(A_{k 1}+e^{i \varphi} A_{k 3}\right) e^{-i E_{k} t} .
$$


Under the unitary evolution of the Hamiltonian $H^{\prime}$, the density of probability $\wp(\mathrm{Bell})$ for finding the entangled Bell state in this coupled QD system is proportional to $\mid\left(\left.\left\langle 0\left|+e^{i \varphi}\langle 2|\right) \mid \Psi(t)\right\rangle_{\Lambda}\right|^{2}\right.$. More explicitly we find

$$
\wp(\text { Bell })=\frac{1}{2}\left|\sum_{k} C_{k}\left(A_{k 1}+e^{i \varphi} A_{k 3}\right) e^{-i E_{k} t}\right|^{2} .
$$

Results and discussion of the time evolution described by Eq. (16), for several different combinations of the physical parameters in the model, are discussed later.

\section{B. Coupling of $\mathrm{N}=3$ QDs and generation of GHZ states}

Here we address the problem of generation of entangled GHZ states of the form $\left|\Psi_{G H Z}\right\rangle=\frac{1}{\sqrt{2}}\left(|000\rangle+e^{i \varphi}|111\rangle\right)$, for any $\varphi$, in the proposed system of 3 coupled QDs. In this case, the Hilbert space $S U(2)^{\otimes 3}$ is spanned by the eight basis vectors associated with the 3 different $J$-subspaces. Without loss of generality, consider the $J=\frac{3}{2}$-subspace as the only one optically active. We introduce the notation $\left|M_{1}\right\rangle \equiv|3 / 2,-3 / 2\rangle \equiv|0\rangle$, $\left|M_{2}\right\rangle \equiv|3 / 2,-1 / 2\rangle \equiv|1\rangle,\left|M_{3}\right\rangle \equiv|3 / 2,1 / 2\rangle \equiv|2\rangle$, and $\left|M_{4}\right\rangle \equiv|3 / 2,3 / 2\rangle \equiv|3\rangle$ to denote the vacuum state, the single-exciton state, the biexciton state and the triexciton state respectively. In the absence of light, the energy levels of the system are given by $E_{0} \equiv E(3 / 2,-3 / 2)=$ $\frac{3}{2}\left(W-\Delta_{\omega}\right), E_{1} \equiv E(3 / 2,-1 / 2)=\frac{1}{2}\left(7 W-\Delta_{\omega}\right), E_{2} \equiv$ $E(3 / 2,1 / 2)=\frac{1}{2}\left(7 W+\Delta_{\omega}\right)$, and $E_{3} \equiv E(3 / 2,3 / 2)=$ $\frac{3}{2}\left(W+\Delta_{\omega}\right)$. We note that, as indicated in the preceding section, the energy separation $E_{3,0} \equiv E_{3}-E_{0}=3 \Delta_{\omega}$ is unaffected by the interdot interaction strength $W$. Now we consider the effect of the pulse of light $\xi(t)$ over this system of 3 QDs in the $J=\frac{3}{2}-$ subspace: the associated Hamiltonian is:

$\widehat{H^{\prime}}=\left(\begin{array}{cccc}\frac{3 W}{2}-\frac{3 \Delta_{\omega}}{2} & \sqrt{3} A^{*} & 0 & 0 \\ \sqrt{3} A & \frac{7 W}{2}-\frac{\Delta_{\omega}}{2} & 2 A^{*} & 0 \\ 0 & 2 A & \frac{7 W}{2}+\frac{\Delta_{\omega}}{2} & \sqrt{3} A^{*} \\ 0 & 0 & \sqrt{3} A^{\frac{3 W}{2}+\frac{3 \Delta_{\omega}}{2}}\end{array}\right)$.

Diagonalization leads us to the following 4 th order equation:

$$
\begin{aligned}
& \left(\left[\frac{3}{2}\left(W-\Delta_{\omega}\right)-E\right]\left[\frac{1}{2}\left(7 W-\Delta_{\omega}\right)-E\right]-3|A|^{2}\right) \times \\
& \left(\left[\frac{3}{2}\left(W+\Delta_{\omega}\right)-E\right]\left[\frac{1}{2}\left(7 W+\Delta_{\omega}\right)-E\right]-3|A|^{2}\right)- \\
& 4|A|^{2}\left(\frac{3}{2}\left(W-\Delta_{\omega}\right)-E\right)\left(\frac{3}{2}\left(W+\Delta_{\omega}\right)-E\right)=0,
\end{aligned}
$$

which is non-trivial to solve analytically in the case of a pulse with arbitrary frequency $\omega$. However, if $\xi(t)$ is applied at resonance $\left(\Delta_{\omega}=0\right)$, we get the following eigenenergies:

$$
\begin{aligned}
& E_{0,1}=\frac{5}{2} W+|A| \pm \sqrt{(W+|A|)^{2}+3|A|^{2}} \\
& E_{2,3}=\frac{5}{2} W-|A| \pm \sqrt{(W-|A|)^{2}+3|A|^{2}}
\end{aligned}
$$

with eigenvectors:

$$
\begin{gathered}
\left|E_{0,1}\right\rangle=\eta_{0,1} \times \\
{\left[|0\rangle+\left(\frac{E_{0,1}-\frac{3 W}{2}}{\sqrt{3}|A|}\right)|1\rangle+\left(\frac{E_{0,1}-\frac{3 W}{2}}{\sqrt{3}|A|}\right)|2\rangle+|3\rangle\right],} \\
\left|E_{2,3}\right\rangle=\eta_{2,3} \times \\
{\left[|0\rangle+\left(\frac{E_{2,3}-\frac{3 W}{2}}{\sqrt{3}|A|}\right)|1\rangle-\left(\frac{E_{2,3}-\frac{3 W}{2}}{\sqrt{3}|A|}\right)|2\rangle-|3\rangle\right],}
\end{gathered}
$$

where the coefficients $\eta_{i}=\frac{1}{\sqrt{2}}\left(1+\frac{\left(E_{i}-\frac{3 W}{2}\right)^{2}}{3|A|^{2}}\right)^{-\frac{1}{2}}$, with $i=0, \ldots, 3$ are normalization constants. The associated total wave function $|\Psi(t)\rangle_{\Lambda}$ (Eq. (14)) depends on the chosen initial condition $|\Psi(t=0)\rangle \equiv\left|\Psi_{0}\right\rangle$ and is a linear combination of the eigenfunctions (20) and (21). We have computed, in both rotating and laboratory frames, the analytical expressions for $|\Psi(t)\rangle_{\Lambda}$ for all of the initial conditions $\left|\Psi_{0}\right\rangle=\{|0\rangle,|1\rangle,|2\rangle,|3\rangle\}$. As an example of this procedure, we give the result for the zero-exciton state as the initial state, i.e. $\left|\Psi_{0}\right\rangle=\left|M_{1}\right\rangle \equiv|0\rangle$. In this case, the wave function $|\Psi(t)\rangle_{\Lambda}$ is spanned by the following coefficients $C_{k}$ and $A_{k j}$ :

$$
\begin{gathered}
C_{0}=\frac{E_{1}-\frac{3 W}{2}}{2 \eta_{0}\left(E_{1}-E_{0}\right)}, C_{1}=\frac{E_{0}-\frac{3 W}{2}}{2 \eta_{1}\left(E_{0}-E_{1}\right)}, \\
C_{2}=\frac{E_{3}-\frac{3 W}{2}}{2 \eta_{2}\left(E_{3}-E_{2}\right)}, C_{3}=\frac{E_{2}-\frac{3 W}{2}}{2 \eta_{3}\left(E_{2}-E_{3}\right)} ; \\
\widehat{A} \equiv\left(\begin{array}{cccc}
\eta_{0} & \frac{\left(E_{0}-\frac{3}{2} W\right) e^{i \varphi}}{\sqrt{3}|A|} \eta_{0} & \frac{\left(E_{0}-\frac{3}{2} W\right) e^{i 2 \varphi}}{\sqrt{3}|A|} \eta_{0} & e^{i 3 \varphi} \eta_{0} \\
\eta_{1} & \frac{\left(E_{1}-\frac{3}{2} W\right) e^{i \varphi}}{\sqrt{3}|A|} \eta_{1} & \frac{\left(E_{1}-\frac{3}{2} W\right) e^{i 2 \varphi}}{\sqrt{3}|A|} \eta_{1} & e^{i 3 \varphi} \eta_{1} \\
\eta_{2} & \frac{\left(E_{2}-\frac{3}{2} W\right) e^{i \varphi}}{\sqrt{3}|A|} \eta_{2} & -\frac{\left(E_{2}-\frac{3}{2} W\right) e^{i 2 \varphi}}{\sqrt{3}|A|} \eta_{2} & -e^{i 3 \varphi} \eta_{2} \\
\eta_{3} & \frac{\left(E_{3}-\frac{3}{2} W\right) e^{i \varphi}}{\sqrt{3}|A|} \eta_{3} & -\frac{\left(E_{3}-\frac{3}{2} W\right) e^{i 2 \varphi}}{\sqrt{3}|A|} \eta_{3} & -e^{i 3 \varphi} \eta_{3}
\end{array}\right) .
\end{gathered}
$$

The density of probability $\wp(G H Z)$ of finding the entangled GHZ state between vacuum and triexciton states is given by:

$$
\wp(G H Z)=\frac{1}{2}\left|\sum_{k} C_{k}\left(A_{k 1}+e^{i \varphi} A_{k 4}\right) e^{-i E_{k} t}\right|^{2} .
$$

Analytical and numerical computations have been performed for all of the initial conditions $\left|\Psi_{0}\right\rangle$ mentioned above. These results enable us to obtain specific conditions for the realization of such maximally entangled 
GHZ states starting from suitable $\varphi$-pulses and experimental parameters $\epsilon, W$, and $A$. Details are discussed in section III.

The quantum dynamical problem given by Eq. (5) is easily expressed in terms of the expansion coefficients $d_{M}(t)$ of the wave function. As usual, we write $|\Psi(t)\rangle_{\Lambda}=\sum_{M=-J}^{J} d_{M}(t) e^{-i E_{M} t}|M\rangle$, so the time dependent problem is reduced to finding the solutions of the following set of $2 J+1$ linear differential equations:

$$
\begin{gathered}
i \partial_{t} d_{M}(t)= \\
A \sqrt{J(J+1)-M(M-1)} e^{\left(E_{M}-E_{M-1}-\omega\right) i t} d_{M-1}(t)+ \\
A^{*} \sqrt{J(J+1)-M(M+1)} e^{\left(E_{M}-E_{M+1}+\omega\right) i t} d_{M+1}(t),
\end{gathered}
$$

where $E_{M}=E(J, M)+\omega$. More explicitly, $E_{M, M-1} \equiv$ $E_{M}-E_{M-1}=\epsilon+W[1-2 M], E_{M, M+1}=W[1+2 M]-$ $\epsilon$, and the problem given by Eq. (25) can be expressed in terms of reduced units as follows:

$$
\begin{gathered}
i \partial_{\tau} f_{M}(\tau)= \\
\lambda \sqrt{J(J+1)-M(M-1)} e^{[1+\mu(1-2 M)-\nu] i \tau} f_{M-1}(\tau)+ \\
\lambda^{*} \sqrt{J(J+1)-M(M+1)} e^{-[1-\mu(1+2 M)-\nu] i \tau} f_{M+1}(\tau),
\end{gathered}
$$

with the dimensionless parameters $\lambda=\frac{A}{\epsilon}, \mu=\frac{W}{\epsilon}$, $\nu=\frac{\omega}{\epsilon}, \tau=\epsilon t$, and $d_{M}(t)=f_{M}(\tau)$. The set of Eqs. (26) gives the dynamics for any number of QDs and $(J, M)$ values. Numerical solutions were found by varying the parameters $\lambda, \mu$, and $\nu$. Comparison between these numerical solutions and the analytical ones yields excellent agreement for the generation of both Bell and GHZ states, starting from suitable initial conditions $\left|\Psi_{0}\right\rangle$. Results will be addressed in the next section.

\section{RESULTS AND DISCUSSION}

In this section we discuss the main results obtained from the computation of both analytical and numerical solutions for the unitary evolution described in the preceding section. Figure 1 shows the probability density for finding the entangled Bell state $(N=2)$ between vacuum and biexciton states given by Eq. (16) as a function of time for the initial condition $\left|\Psi_{0}\right\rangle=|0\rangle$. As seen from Fig. 1, selective pulses of length $\tau_{B}$ can be used to create maximally entangled Bell states in the system of two coupled QDs. The energy $W$ is kept fixed while the amplitude of the radiation pulse $A$ is varied. The results indicate that the time $\tau_{B}$ is increased with diminishing incident field strength $A$. As an example, we consider wide-gap semiconductor QDs, like ZnSe based QDs, with band gap $\epsilon=2.8 \mathrm{eV}$ and resonant optical frequency $\omega=4.3 \times 10^{15} \mathrm{~s}^{-1}$. For a 0 or $2 \pi$-pulse, $W=0.1$ and $A=\frac{1}{25}$, Fig. 1(a) shows that the generation of the state $\frac{1}{\sqrt{2}}(|0\rangle+|2\rangle)$ requires a pulse of length
$\tau_{B}=7.7 \times 10^{-15} \mathrm{~s}$. By changing the value of the parameter $A$ (see Figs. 1(b), (c) and (d)), we can modify the length $\tau_{B}$ of this Bell pulse (Fig. 1 covers the interval $\left.10^{-11} \mathrm{~s}<\tau_{B}<10^{-15} \mathrm{~s}\right)$. Another method for manipulating the length $\tau_{B}$ is shown in Fig. 2. Here we vary $W$ for fixed $A=10^{-3}$. Experimentally, this variation of $W$ can be tailored by changing the interdot distance. In this case, the analysis shows that for a fixed value of $A$ the length $\tau_{B}$ decreases with decreasing interaction strength $W$.

The same investigation of parameter dependance was performed for the case of the entangled GHZ state $(N=$ 3 ) between vacuum and triexciton states. We calculate the probability given by Eq. (24) as a function of time, starting with the initial condition $\left|\Psi_{0}\right\rangle=|0\rangle$. Figure 3 shows the selective pulses used to create such maximally entangled GHZ states in the system of three coupled QDs. For example, in the case of Fig. 3(a), the generation of the GHZ state $\frac{1}{\sqrt{2}}(|0\rangle+|3\rangle)$ requires a time $\tau_{G H Z}=1.3 \times 10^{-14} \mathrm{~s}$. Figures $3(\mathrm{~b}),(\mathrm{c})$, and (d) explore several different ranges for the $\tau_{G H Z}$-pulses required in the generation of such GHZ states. For fixed $W$, the time $\tau_{G H Z}$ increases with decreasing incident field strength $A$. In contrast, for fixed $A$, the length $\tau_{G H Z}$ decreases with decreasing interdot interaction strength.

The above results are not restricted to $\mathrm{ZnSe}$-based QDs: by employing semiconductors of different bandgap $\epsilon$ (e.g. GaAs), other regions of parameter space can be explored. We have studied the time evolution of the system of QDs for several different values of the phase $\varphi$. These give similar qualitative results to the ones discussed previously. Here we only include the 0 or $2 \pi$-pulse results since these are ones corresponding to the discussion in Sec. IV. The relevant experimental conditions as well as the required coherent control to realize the above combinations of parameters, are compatible with those demonstrated in Ref. 113. We point out that the procedure described in this paper is valid for any value of the phase constant $\varphi$, in contrast to Ref. [21] where analytic results were derived for the particular case $\varphi=\frac{\pi}{2}$. The generation of maximally entangled states in this paper has considered the experimental situation of global laser pulses only; however, by using near-field optical spectroscopy [15, individual QDs from an ensemble can be addressed by using local pulses, a feature that can be exploited to generate entangled states with different symmetries, such as the antisymmetric state $\frac{1}{\sqrt{2}}(|01\rangle-|10\rangle)$. We will hence be able to generate the complete Bell basis consisting of four mutually orthogonal states for the 2 qubits, all of which are maximally entangled, i.e. the set of states $\frac{1}{\sqrt{2}}\{(|00\rangle+|11\rangle),(|00\rangle-|11\rangle),(|01\rangle+|10\rangle),(|01\rangle-|10\rangle)\}$. From a general point of view, this basis is of fundamental relevance for quantum information processing. We stress that the optical generation of excitonic entangled states 
in coupled QDs given here could be exploited in solid state devices to perform quantum protocols, as recently proposed in Ref. [26] for teleporting an excitonic state in a coupled QD system.

\section{QUANTUM INFORMATION PROCESSING IN COUPLED DOTS}

To perform quantum computation operations, we can use an initial pure state followed by a series of transformations on this state using unitary operations. Another possibility is to use an initial mixed state, providing the decoherence time is sufficiently long [10]. In order to implement such quantum operations we need two elements: the Hadamard transformation and the quantum CNOT gate. In the orthonormal computation basis of single qubits $\{|0\rangle,|1\rangle\}$, the CNOT gate acts on two qubits $\left|\varphi_{i}\right\rangle$ and $\left|\varphi_{j}\right\rangle$ simultaneously as follows: $\mathrm{CNOT}_{i j}\left(\left|\varphi_{i}\right\rangle\left|\varphi_{j}\right\rangle\right) \mapsto\left|\varphi_{i}\right\rangle\left|\varphi_{i} \oplus \varphi_{j}\right\rangle$. Here $\oplus$ denotes $a d-$ dition modulo 2, and the indices $i$ and $j$ refer to the control bit and the target bit respectively. The Hadamard transformation $H^{T}$ acts only on single qubits by performing the rotations: $H^{T}(|0\rangle) \mapsto \frac{1}{\sqrt{2}}(|0\rangle+|1\rangle)$, and $H^{T}(|1\rangle) \mapsto \frac{1}{\sqrt{2}}(|0\rangle-|1\rangle)$. In our scheme, $|0\rangle$ represents the vacuum state for excitons while $|1\rangle$ represents a single exciton. Experimental demonstration of single qubit rotations (and hence the Hadamard transformation) in the case of individual excitons confined to QDs should now be possible, as a result of a recent experiment reporting direct observation of excitonic Rabi oscillations in semiconductor quantum wells [27]. Despite the fact that Rabi Flopping in QDs is still under intensive experimental study, results given in Ref. [27] lead us to believe that we are not too far away from the experimental observation of such excitonic Rabi oscillations in QDs and hence the demonstration of single qubit operations.

The adequate preparation, computation and readout of information, in addition to the coherent coupling of the qubits to the environment, are compulsory steps for the successful construction of a universal quantum computer. We briefly review these requirements and their relationship with the model proposed here: (1) A very well defined Hilbert space: We must have an adequate control over the Hilbert space of qubits. In our scheme, the orthonormal computation basis of single qubits is represented by the vacuum state $(|0\rangle)$ and the single state of excitons $(|1\rangle)$. (2) Initializing the computer: Before commencing any quantum computation task, we need a rapid relaxation of our qubits to their ground state, i.e. zero excitons per dot. In our case, numerical values indicate that this state is easily achieved by turning the laser off and waiting for a few femtoseconds. (3) Inputting initial data and readout: As pure and entangled states with different symmetries can be obtained using the experi- mental techniques described in Ref. 13 15, we would have the ability to manipulate the input of the quantum state of the QD system. Hence, we would have the experimental possibility to control the optical excitation and to detect individual QD signals from an entire dot ensemble, thereby facilitating individual qubit control for the readout. In fact, nanoprobing enables us to measure directly the excitonic and biexcitonic luminescence from single QDs [15]. (4) Universal set of gate operations: We need to be able to perform single qubit rotations and two qubit gates. We stress that the generation of the maximally entangled states shown in Figs. $1-3$ corresponds to the physical realization of a Hadamard transformation followed by a CNOT operation in the Bell case, and two CNOT operations in the GHZ case. As to the practical semiconductor nanostructure implementation, this set of gate operations is in a preliminary stage of investigation and demands intensive experimental study. (5) Decoherence and the coupling to the environment: By taking into account decoherence mechanisms (exciton-acousticphonon type) on the process of generation of the entangled states discussed here, a recent work by Rodríguez et al. 228] has shown that this generation is preserved over a reasonable parameter window hence giving the possibility of performing the unitary transformations required for quantum computing before decoherence of the excitonic states takes place.

\section{CONCLUSIONS}

In summary, we have solved both analytically and numerically the quantum mechanical equation-of-motion for excitons in two and three coupled QD systems driven by classical pulses of light. By doing this, we have been able to provide a mechanism for preparing maximally entangled Bell and GHZ states via excitons in optically-driven QDs, exploiting current levels of coherent optical control such as the ones demonstrated using ultra-fast spectroscopy [13,14 and near-field optical spectroscopy [15]. This mechanism enables us to generate single qubit rotations, such as the Hadamard one, and quantum CNOT gates. In particular, the procedure presented here leads us to the generation of the whole Bell basis, a fact that can be exploited in the process of quantum teleportation of excitonic states in systems of coupled QDs [26]. Furthermore, by taking into account the main decoherence mechanisms, e.g. exciton-acousticphonon, we find that this optical generation of quantum entanglement is preserved over a reasonable parameter window. This leads to the possibility of performing several hundred quantum computation operations before decoherence of these excitonic states takes place. 


\section{ACKNOWLEDGMENTS}

J.H.R. and L.Q. acknowledge the support of COLCIENCIAS. J.H.R. thanks D.J.T. Leonard for helpful discussions, and the hospitality of the ESF-QIT programme meeting 1999 in Cambridge, where part of this work was performed.

※ j.reina-estupinan@physics.ox.ac.uk

† luis@anacaona.uniandes.edu.co

¥ n.johnson@physics.ox.ac.uk

[1] R.P. Feynman, Int. J. Theor. Phys. 21, 467 (1982), Opt. News 11, 11 (1985).

[2] D. Deutsch, Proc. R. Soc. London A 400, 97 (1985).

[3] W.K. Wooters and W.H. Zurek, Nature 299, 802 (1982); N.D. Mermin, Physics Today 38 4, 38 (1985); W.H. Zurek, ibid. 44 10, 36 (1991); C.H. Bennett, ibid. 48 10, 24 (1995); see articles in the special issue on quantum information, Physics World, March 1998; A. Ekert and R. Jozsa, Rev. Mod. Phys. 68, 733 (1996).

[4] A.K. Ekert, Phys. Rev. Lett. 67, 661 (1991); C.H. Bennett, G. Brassard, and N.D. Mermin, ibid. 68, 557 (1992); C.H. Bennett, G. Brassard, and A. Ekert, Scientific American 267 4, 26 (1992).

[5] C.H. Bennett, G. Brassard, C. Crépeau, R. Jozsa, A. Peres, and W.K. Wooters, Phys. Rev. Lett. 70, 1895 (1993).

[6] P.W. Shor, in Proceedings of the 35th Annual Symposium on the Foundations of Computer Science, ed. by S. Goldwasser (IEEE Computer Society, Santa Fe, Los Alamitos, CA), 124 (1994).

[7] P.W. Shor, Phys. Rev. A 52, 2493 (1995); A.M. Steane, Phys. Rev. Lett. 77, 793 (1996).

[8] J.I. Cirac and P. Zoller, Phys. Rev. Lett. 74, 4091 (1995); C. Monroe, D.M. Meekhof, B.E. King, W.M. Itano, and D.J. Wineland, ibid. 75, 4714 (1995).

[9] Q.A. Turchete, C.J. Hood, W. Lange, H. Mabuchi, and H.J. Kimble, Phys. Rev. Lett. 75, 4710 (1995).

[10] N.A. Gershenfeld and I.L. Chuang, Science 275, 350 (1997); D.G. Cory, A.F. Fahmy, and T.F. Havel, Proc. Natn. Acad. Sci. USA 94, 1634 (1997); E. Knill, I.L. Chuang, and R. Laflamme, Phys. Rev. A 57, 3348 (1998); J.A. Jones, M. Mosca, and R.H. Hansen, Nature 393, 344 (1998).

[11] A. Shnirman, G. Schön, and Z. Hermon, Phys. Rev. Lett. 79, 2371 (1997); D.V. Averin, Solid State Commun. 105, 659 (1998); Y. Makhlin, G. Schön, and A. Shnirman, Nature 398, 305 (1999).

[12] B.E. Kane, Nature 393, 133 (1998); D. Loss, and D.P. DiVincenzo, Phys. Rev. A 57, 120 (1998); G. Burkard, D. Loss, and D.P. DiVincenzo, Phys. Rev. B 59, 2070 (1999); A. Barenco, D. Deutsch, A. Ekert, and R. Jozsa, Phys. Rev. Lett. 74, 4083 (1995); A. Imamoglu, D.D. Awschalom, G. Burkard, D.P. DiVincenzo, D. Loss, M. Sherwin, and A. Small, Phys. Rev. Lett. 83, 4204 (1999); R. Vrijen, E. Yablonovitch, K. Wang, H.W. Jiang, A. Balandin, and D.P. DiVincenzo, quant-ph/9905096.
[13] N.H. Bonadeo, J. Erland, D. Gammon, D.S. Katzer, D. Park, and D.G. Steel, Science 282, 1473 (1998).

[14] N.H. Bonadeo, G. Chen, D. Gammon, D.S. Katzer, D. Park, and D.G. Steel, Phys. Rev. Lett. 81, 2759 (1998).

[15] A. Chavez-Pirson, J. Temmyo, H. Kamada, H. Gotoh, and H. Ando, Appl. Phys. Lett. 72, 3494 (1998).

[16] J.S. Bell, Physics 1, 195 (1964).

[17] A. Einstein, B. Podolsky, and N. Rosen, Phys. Rev. 47, 777 (1935).

[18] D.M. Greenberger, M.A. Horne, and A. Zeilinger, in Bell's Theorem, Quantum Theory and Conceptions of the Universe, ed. M. Kafatos, pp. 73, Kluwer, Dordrecht, The Netherlands (1989); D.M. Greenberger, M.A. Horne, A. Shimony, and A. Zeilinger, Am. J. Phys. 58, 1131 (1990).

[19] A. Aspect, J. Dalibard, and G. Roger, Phys. Rev. Lett. 49, 1804 (1982).

[20] Throughout this paper we refer to this phase factor as the " $\varphi$-pulse", to denote the type of entangled state generated in the optical process.

[21] L. Quiroga and N.F. Johnson, Phys. Rev. Lett. 83, 2270 (1999).

[22] We ignore the constant term $\frac{W}{2} \sum_{p}\left\{c_{p}^{\dagger} c_{p}+h_{p} h_{p}^{\dagger}\right\}=\frac{W N}{2}$ in the Hamiltonian $H$.

[23] In general for $J=\frac{N}{2}$ the multiplicity $D_{J}=1$, hence $q$ is irrelevant.

[24] J.H. Reina, Clarendon Laboratory Report (unpublished).

[25] Write $|\Psi(0)\rangle=\sum_{k} \beta_{k}\left|M_{k}\right\rangle \quad\left(\beta_{k}=\left\langle M_{k} \mid \Psi(0)\right\rangle\right)$. From the expansion given for $|\Psi(t)\rangle_{\Lambda}$ it follows that $|\Psi(0)\rangle=\sum_{k} C_{k}\left|\psi_{k}\right\rangle$, hence the general expresion for the coefficients $C_{k}$ becomes $C_{k}=\left\langle\psi_{k} \mid \Psi(0)\right\rangle=$ $\sum_{j} \beta_{j}\left\langle\psi_{k} \mid M_{j}\right\rangle=\sum_{j} \beta_{j} A_{k j}^{*}$.

[26] J.H. Reina and N.F. Johnson, cond-mat/9906034.

[27] A. Schülzgen, R. Binder, M.E. Donovan, M. Lindberg, K. Wundke, H.M. Gibbs, G. Khitrova, and N. Peyghambarian, Phys. Rev. Lett. 82, 2346 (1999).

[28] F.J. Rodríguez, L. Quiroga, and N.F. Johnson, condmat/9909139

FIG. 1. Generation of the Bell State $\frac{1}{\sqrt{2}}(|00\rangle+|11\rangle)$. These pulses correspond to the realization of the Hadamard gate followed by a quantum CNOT gate. $W=0.1, \varphi=0$, and (a) $A=\frac{1}{25}$, (b) $A=\frac{1}{50}$, (c) $A=10^{-2}$, and (d) $A=10^{-3}$. In the Figures $1-3,|\Psi(t)\rangle$ denotes the total wavefunction of the system at time $t$ in both laboratory (solid curves) and rotating frames (dashed curves). The energy is in units of the band gap $\epsilon$, and $\left|\Psi_{0}\right\rangle=|0\rangle$ in all of the figures.

FIG. 2. Generation of the Bell state $\frac{1}{\sqrt{2}}(|00\rangle+|11\rangle)$. $A=10^{-3}, \varphi=0$, and (a) $W=0.1$, (b) $W=0.05$, and (c) $W=10^{-2}$.

FIG. 3. Generation of the GHZ state $\frac{1}{\sqrt{2}}(|000\rangle+|111\rangle)$. These pulses correspond to the realization of the Hadamard gate followed by two quantum CNOT gates. $W=0.1, \varphi=0$, and (a) $A=\frac{1}{25}$, (b) $A=\frac{1}{50}$, (c) $A=10^{-2}$, and (d) $A=10^{-3}$. 
Figure 2

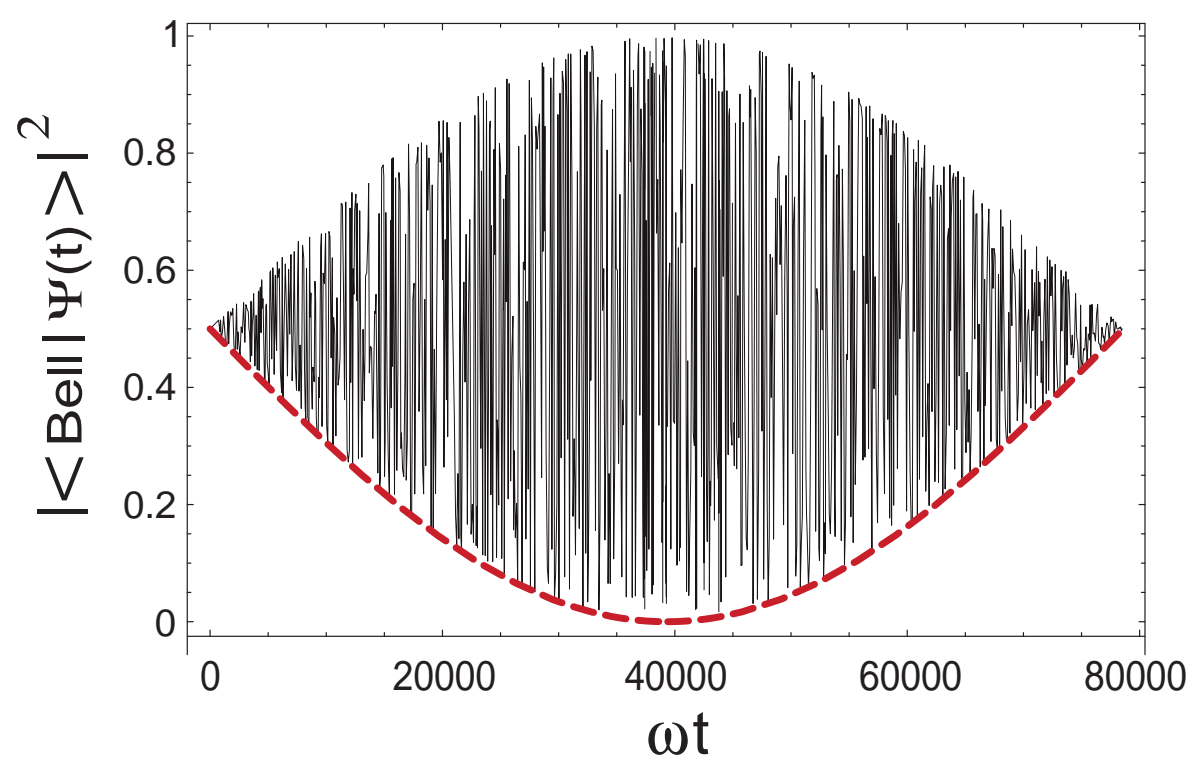

(a)

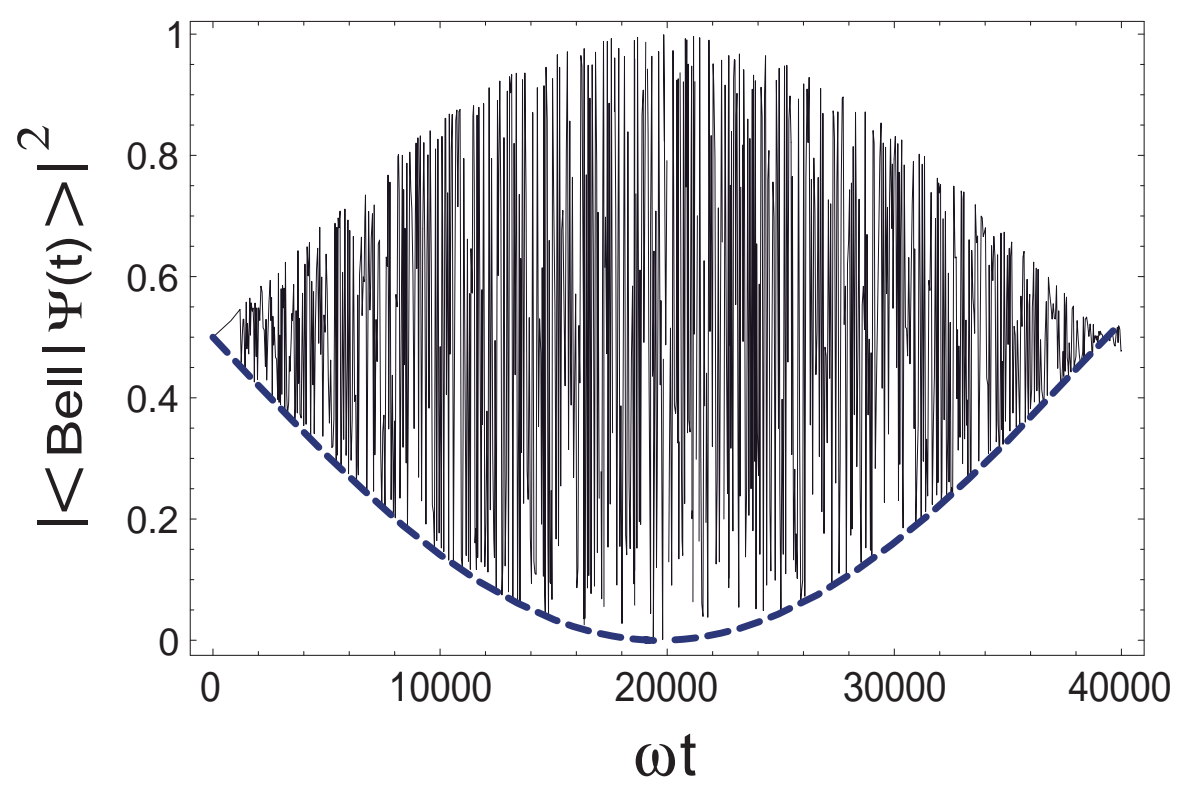

(b)

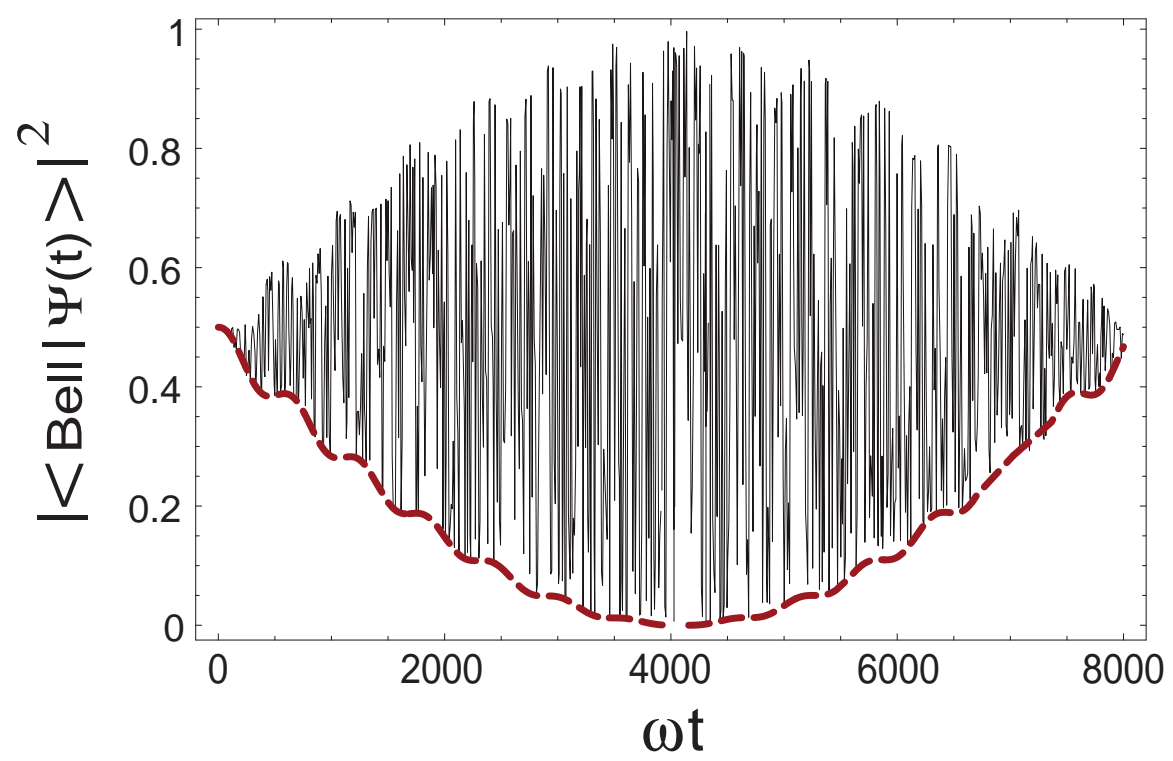

(c) 
Figure 3

(a)

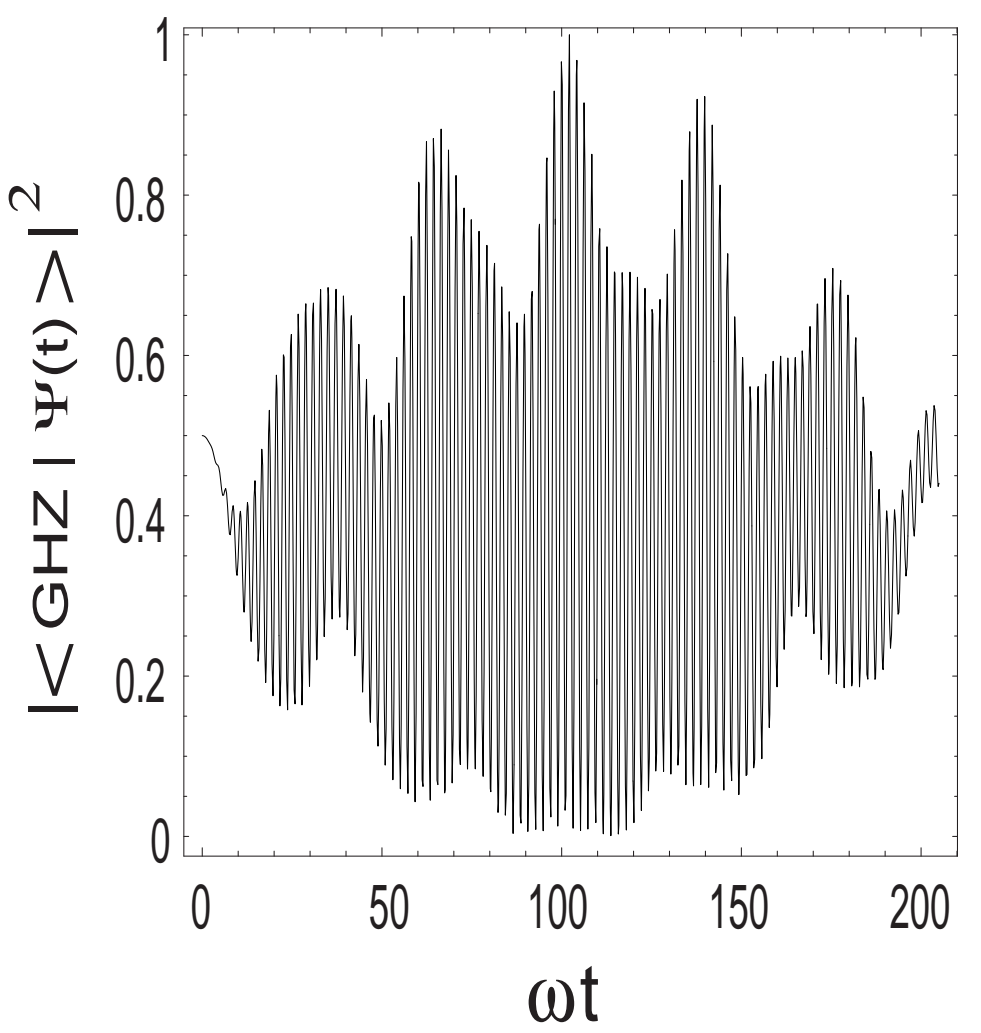

(c)

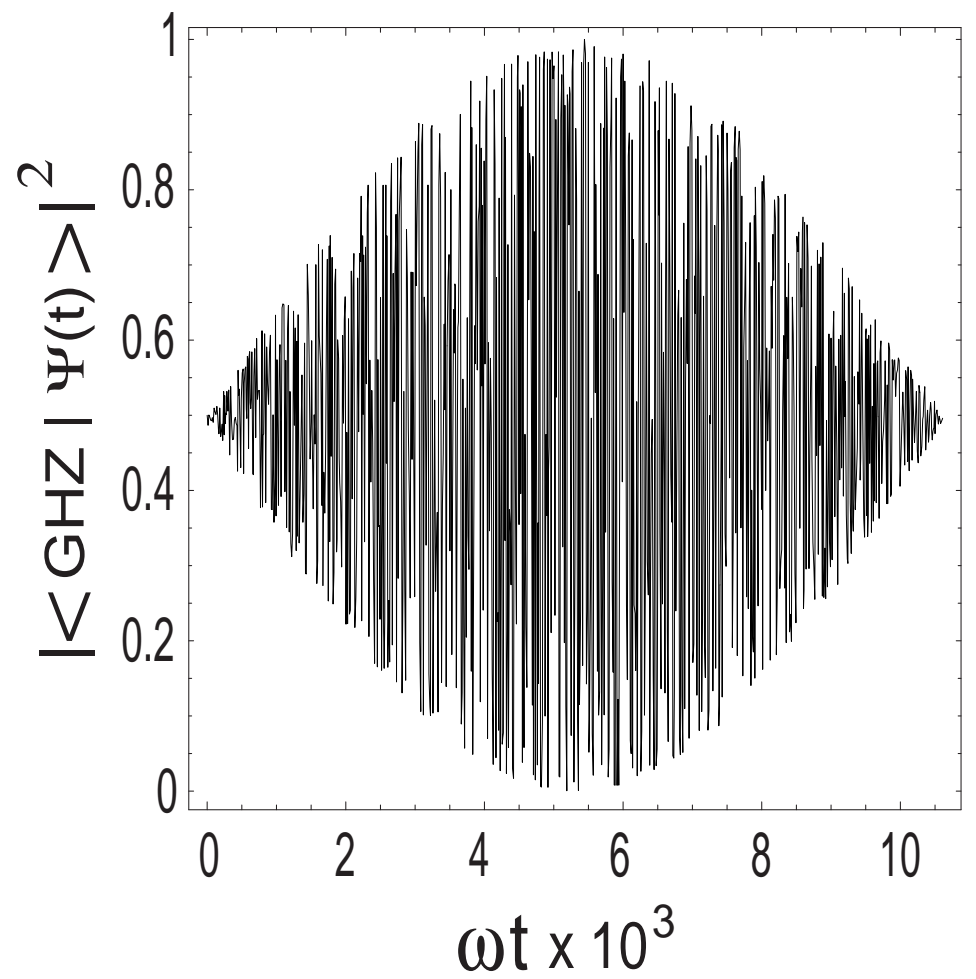

(b)

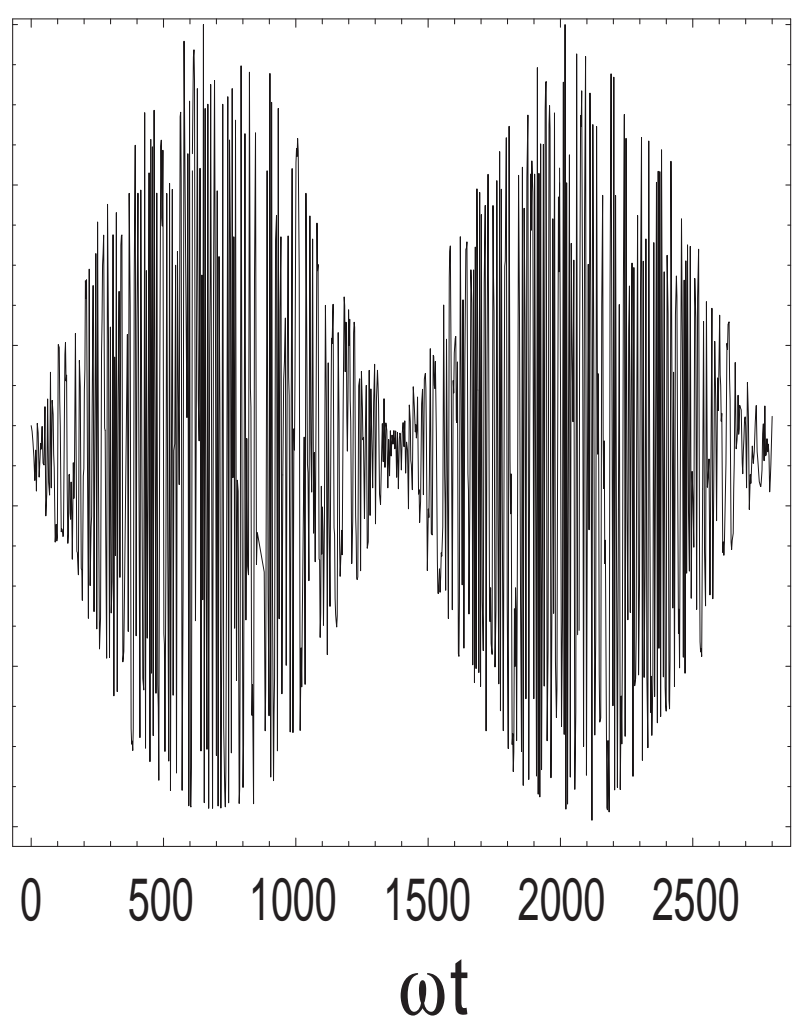

(d)

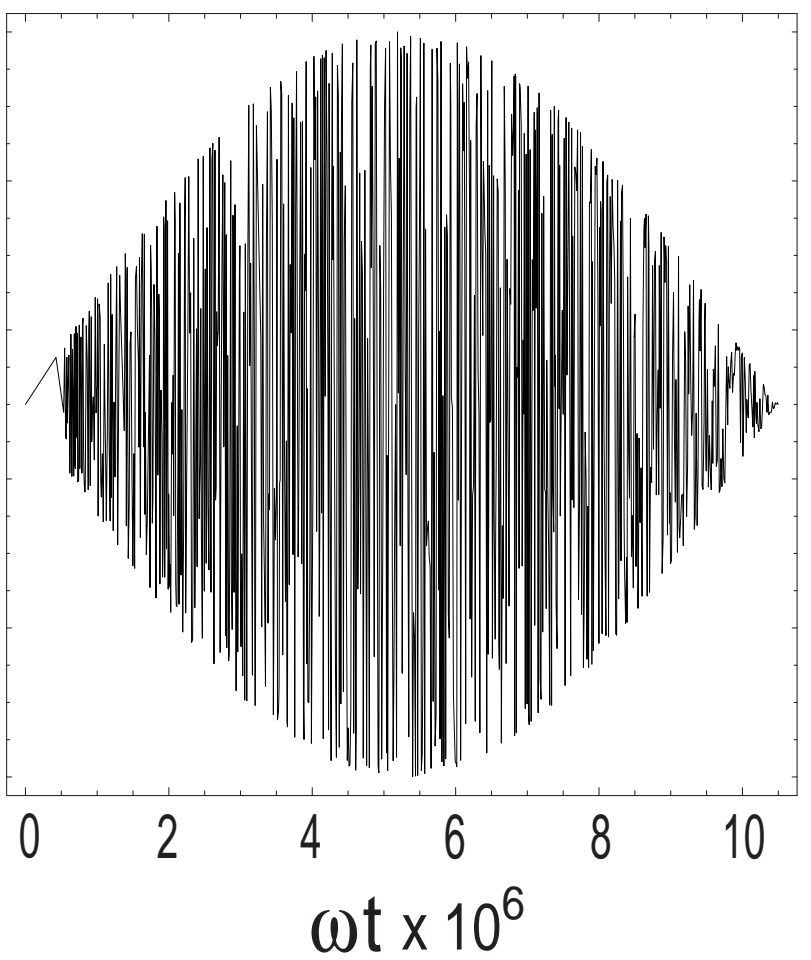

